\title{
Abordagens teóricas sobre o relacionamento entre empresas e universidades e o cenário brasileiro
}

\author{
Julia Paranhos* \\ Lia Hasenclever ${ }^{\dagger}$ \\ Fernanda Steiner Perin $\ddagger$
}

\begin{abstract}
Resumo
O objetivo deste artigo é apresentar as características de três abordagens sobre o relacionamento entre empresas e universidades. No modelo da hélice tríplice, a universidade adquire a função de comercialização do conhecimento, assim sendo vista como um ator do desenvolvimento econômico. Na abordagem de sistemas de inovação, a universidade cumpre o papel de formadora de recursos humanos e de parceira das empresas para gerar inovação. E, na abordagem latinoamericana, a universidade tem uma função importante no desenvolvimento social, pois estaria alinhada às necessidades dos países periféricos. Em cada uma destas visões, a universidade e a empresa possuem papéis e formas de interação diferenciados. No Brasil, foi visto que os incentivos para fomentar o papel da universidade no desenvolvimento seguiram as orientações das abordagens teóricas mencionadas, mas acabaram por ficar deslocadas da realidade. Desta forma, os resultados decorrentes são incipientes e pouco satisfatórios até o momento.
\end{abstract}

Palavras-chave: relacionamento empresa-universidade, hélice tríplice, sistemas de inovação, América Latina, Brasil.

JEL: O30, O32, O38

\section{Introdução}

Novas teorias, abordagens e propostas de interação entre empresas e universidades foram desenvolvidas nas últimas décadas com o objetivo de acelerar o desenvolvimento cada vez mais baseado em conhecimento. Algumas destas enfatizam os novos papéis da

\footnotetext{
* Professora adjunta do Instituto de Economia da Universidade Federal do Rio de Janeiro (IE/UFRJ). Email: juliaparanhos@ie.ufrj.br.

${ }^{\dagger}$ Professora colaboradora do Instituto de Economia da Universidade Federal do Rio de Janeiro (IE/UFRJ) e da Universidade Cândido Mendes de Campos dos Goytacazes (UCAM). E-mail: lia@ie.ufrj.br.

‡Doutoranda do Programa de Pós-Graduação em Economia do Instituto de Economia da Universidade Federal do Rio de Janeiro (PPGE/IE/UFRJ). E-mail: fernanda.steinerperin@gmail.com.
} 

brasileiro

universidade, como, por exemplo, o de ator do desenvolvimento econômico e criador de empresas - abordagem da hélice tríplice -, da universidade como ator do desenvolvimento social - abordagem latino-americana, e outras que reservam um papel para a universidade como formadora de recursos humanos qualificados e parceira das empresas na inovação - abordagem de sistemas de inovação -, e as consideram como coadjuvantes no processo de inovação, que por sua vez é considerado o principal processo de concorrência entre as empresas.

Em cada uma destas visões, a universidade e a empresa desempenham um papel diferenciado, e a interação entre elas é observada e analisada diferentemente. No entanto, em todas as abordagens a criação do conhecimento se dá de forma interativa, complexa, e multidisciplinar, ou seja, pelo modo 2 de criação do conhecimento ${ }^{1}$, segundo Gibbons et al. (1994).

O objetivo deste artigo é apresentar as principais características destas três abordagens sobre o relacionamento entre empresas e universidades, e identificar quais as principais formas de atuação das universidades em prol do desenvolvimento econômico e social, previstas na literatura sobre o tema.

O estudo foi feito a partir de uma revisão bibliográfica em periódicos e livros sobre as abordagens do relacionamento universidade-empresa, e sobre sua interação no Brasil, baseado em Paranhos $(2010)^{2}$. Analisa-se, neste artigo, o cenário brasileiro à luz das três abordagens, e discute-se as limitações existentes nesse cenário para a implementação de políticas que ampliem o papel da universidade na inovação e na resolução dos problemas sociais. Para tanto, foram também levantados dados secundários do Instituto Brasileiro de Geografia e Estatística (IBGE), Conselho Nacional de Pesquisa Científica e Tecnológica $(\mathrm{CNPq})$, e da Associação Nacional de Entidades Promotoras de Empreendimentos Inovadores (Anprotec).

O artigo está dividido em quatro seções além desta Introdução e da Conclusão. Nas três primeiras seções são apresentadas as características de cada abordagem com a seguinte ordem: hélice tríplice; sistemas de inovação; e latino-americana. O foco dessas três seções é destacar os diferentes papéis exercidos pela universidade na produção e difusão do conhecimento e suas distintas formas de interação com a sociedade. Na quarta seção, a título de discussão do tema que as três abordagens suscitam, observa-se como a literatura tem influenciado a realidade brasileira da relação entre as universidades e os demais atores no estabelecimento de marcos normativo, e os resultados que estes marcos têm tido em ampliar a interação dos atores no sistema nacional de inovação brasileiro.

\footnotetext{
${ }^{1}$ Gibbons et al. (1994) definem que a criação do conhecimento é realizada de duas formas. No Modo 1, a criação do conhecimento ocorre sem objetivo prévio de aplicação prática e envolve apenas uma área do conhecimento. Desta forma, é insuficiente para atender uma demanda tecno-científica das empresas e da sociedade nos dias atuais. Por outro lado, no Modo 2, a criação do conhecimento é multidisciplinar e busca por um resultado pré-definido, sendo mais próxima das necessidades sociais e econômicas

${ }^{2}$ Publicado em formato de livro em Paranhos (2012)
}

Econômica-Niterói, v. 20, n. 1, p. 9-29. Junho, 2018 


\section{A universidade como ator do desenvolvimento econômico - hélice tríplice e a comercialização do conhecimento}

O modelo da hélice tríplice ${ }^{3}$ proposto por Etzkowitz e Leydesdorff (2000) é uma forma de representar o sistema de inovação e seu funcionamento a partir da interação entre três esferas institucionais - universidade, indústria e governo. Cada uma destas esferas representa uma hélice que interage em três dimensões com as demais Etzkowitz (2002). A primeira dimensão ocorre no interior de cada hélice com a transformação de cada um dos atores; podem-se citar, como exemplos, as alianças estratégicas entre as empresas, e a inclusão de uma nova missão da universidade - comercialização do conhecimento.

A segunda dimensão é a influência de uma hélice sobre a outra, sinalizando o início da interação entre os atores de diferentes dimensões institucionais para a geração da inovação. Um exemplo desta segunda dimensão seria a aprovação do governo da legislação que estimula a transferência de tecnologia das universidades para as empresas. A terceira dimensão é a criação de instituições trilaterais híbridas oriundas da interação entre as três hélices, com objetivo de apresentar propostas de desenvolvimento de alta tecnologia, como por exemplo centros tecnológicos, incubadoras de empresas, universidades corporativas, instituições governamentais de venture capital.

A dinâmica da hélice tríplice está baseada na auto-organização da produção do conhecimento e troca de informações (com incerteza), e na seleção destas informações dentro de cada subdinâmica existente em cada uma das hélices. O sistema de inovação é conduzido por várias subdinâmicas em várias dimensões, sendo o objetivo do modelo estudar tais dimensões e subdinâmicas. Leydesdorff (2006) identifica as funções de cada uma das subdinâmicas da hélice tríplice: (1) geração de riqueza na economia pela indústria; (2) produção de novidade pelas instituições de Ciência \& Tecnologia (C\&T); (3) controle normativo das demais funções para manutenção e reprodução do sistema.

Em suma, o modelo prevê o relacionamento entre três esferas institucionais de forma equilibrada, que atuam tanto de forma independente, como sobrepostas e em conjunto. A interação universidade-indústria-governo requer novas formas de aprendizado, comunicação e rotinas diferentes por parte das três esferas. A Figura 1 mostra de forma esquemática as dimensões da interação supracitada, que tem como objetivo a criação de um ambiente propício à inovação para aceleração da produção e difusão do conhecimento.

Para Etzkowitz (2002), a geração de inovações por parte da empresa é limitada quanto ao seu grau de novidade, restringindo-se às inovações incrementais. A inovação radical vem de fora da empresa, muitas vezes de outra esfera institucional, como a universitária, onde o foco da pesquisa está no desenvolvimento de quebra de trajetórias científicas e/ou tecnológicas.

\footnotetext{
${ }^{3}$ A intenção nesta seção é apresentar o conceito de Hélice Tríplice, e não explorar todas as variantes do modelo encontrado em Etzkowitz e Leydesdorff (2000)
} 
Figura 1 - Modelo da hélice tríplice

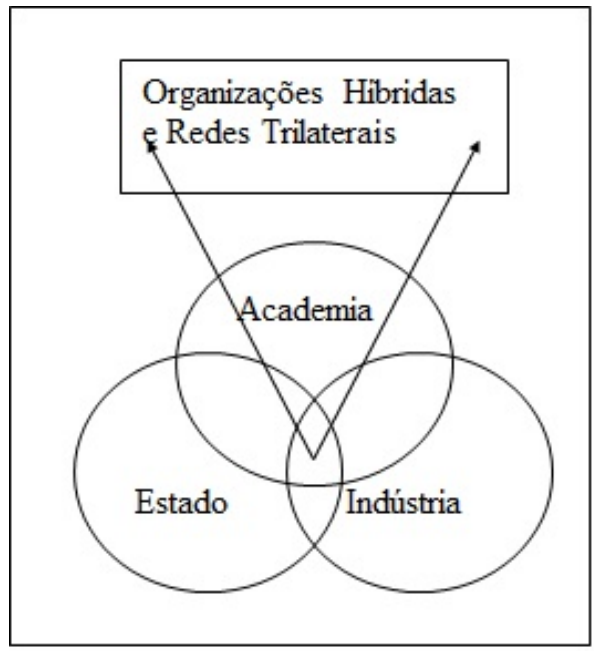

Fonte: Etzkowitz e Leydesdorff (2000)

As relações laterais de parceria entre os atores ganham importância frente às estruturas hierárquicas no desenvolvimento de inovações, permitindo a introdução de expertise de uma esfera social em outra. Neste movimento pode haver a emergência de conflitos de interesse, mas que são vistos como presságio da invenção de papéis inovadores e novos designs organizacionais. Além disso, podem ser introduzidas ideias de uma esfera para outra, ocasionando projetos colaborativos e promovendo a compreensão entre instituições.

A proposta do modelo é que haja circulação de pessoas dentro das hélices (circulação vertical) e entre as hélices (circulação horizontal). Exemplos desta circulação seriam pessoas com prática na indústria atuando como professores e/ou pesquisadores nas universidades, e pesquisadores e professores universitários atuando na indústria ou ainda no governo. A circulação entre as hélices permite o compartilhamento do conhecimento existente e a absorção de novos, porém sem o indivíduo sair totalmente da sua hélice original. Tal circulação pode ocorrer em tempo parcial ou em um período limitado segundo Etzkowitz (2009).

O modelo da hélice tríplice identifica a universidade como o principal ator para a geração de inovação em uma sociedade baseada no conhecimento. Em outras palavras, a universidade passa a ter uma atuação pró-ativa na transferência do conhecimento para geração de inovações e transferência dos recursos humanos treinados para atuarem também em empreendimentos, não se limitando a ser uma simples produtora de conhecimento.

A partir da aproximação entre o setor acadêmico e empresarial, a função da pesquisa básica mudou. De uma pesquisa de longo prazo e sem objetivo de alcançar resultados práticos ("endless frontier") para uma pesquisa ligada à utilização prática dos resultados 
(“endless transition"), (ETZKOWITZ; LEYDESDORFF, 2000). Os grupos de pesquisa universitários passam a atuar como "quase-firmas", (ETZKOWITZ, 2003), mesmo antes de engajarem-se nas atividades empreendedoras propriamente ditas, com atuação direta no setor empresarial na prestação de serviços e realização de parcerias.

As universidades passaram a atuar na "capitalização do conhecimento" e a envolveremse com transferência de tecnologia e formação de empresas, tornando-se universidades empreendedoras. A produção do conhecimento passa a ser um empreendimento econômico. Há, no entanto, cinco normas para a formação de uma universidade empreendedora: capitalização do conhecimento, interdependência em relação às demais esferas, independência institucional, hibridização organizacional para conciliar interdependência e independência, e reflexividade devido à contínua renovação da estrutura interna, (ETZKOWITZ, 2009).

O empreendedorismo, como missão acadêmica, é integrado ao ensino e à pesquisa. São criadas empresas spin-offs geridas por alunos e/ou professores a partir de novos conhecimentos criados pelas necessidades do mercado e pela capacitação interna da universidade (modelo interativo de inovação) ${ }^{4}$. A universidade empreendedora torna-se uma incubadora natural de um ambiente propício à criação de novas áreas científicas interdisciplinares, e de novos setores industriais, (ETZKOWITZ, 2002, 2009).

As universidades empreendedoras têm como características fundamentais: 1) a liderança acadêmica capaz de formular e implementar uma visão estratégica; 2) o controle jurídico sobre os recursos acadêmicos, incluindo a propriedade intelectual que resulta da pesquisa; 3) a capacidade organizacional para transferir tecnologia através de patenteamento, licenciamento e incubação; 4) a característica empreendedora comum a administradores, corpo docente e estudantes. Dadas estas propriedades, passam a ser reconhecidas como fonte de tecnologia, além de fornecedoras de recursos humanos e conhecimento, segundo Etzkowitz (2009).

O estímulo ao aumento da interação entre o setor acadêmico e empresarial passa pela criação de mecanismos de facilitação de transferência de conhecimento. Nas universidades, segundo Etzkowitz (2003), a principal interface com o setor empresarial eram os escritórios de transferência de tecnologia (ETT) ${ }^{5}$ que são o braço do mercado na universidade, ampliando a comercialização do conhecimento acadêmico. Os ETTs funcionam como mecanismo de busca de fontes apropriadas para a transferência do conhecimento o que reduz a incerteza para as empresas, mecanismo de transporte do conhecimento gerando capital social e eficientes mecanismos de busca - e mecanismo de interação entre grupos de diferentes áreas - ampliando as possibilidades de colaboração.

No entanto, uma questão de grande debate que serve de freio a uma maior interação entre universidades e empresas é a questão da apropriação do conhecimento gerado pela

\footnotetext{
${ }^{4}$ Ver Etzkowitz (2003)

${ }^{5}$ No Brasil, após a Lei de Inovação (10.973/2004), as ICTs reuniram os ETTs nos Núcleos de Inovação Tecnológica (NITs).
} 

brasileiro

universidade. Por um lado, argumenta-se que a interação entre o setor acadêmico e empresarial pode ser altamente benéfica para o desenvolvimento econômico de uma sociedade como um todo, porém as empresas não teriam interesse em financiar e apoiar pesquisas que gerem conhecimentos que poderão ser utilizados por suas concorrentes. A parceria empresa-universidade teria então que implicar em exclusividade do conhecimento, pelo menos no primeiro momento, para a empresa parceira. Nesse sentido, a abordagem defende a necessidade de uso de mecanismos de apropriação pela universidade que definam a aplicabilidade do conhecimento para facilitar a sua transferência, como, por exemplo, patente $^{6}$, marca, segredo industrial, etc.

Por outro lado, críticos a essa abordagem defendem a universalidade do conhecimento acadêmico, que deve ser livre e disponível para toda a sociedade, principalmente quando se trata de universidades públicas. O conhecimento deve fluir livremente da esfera universitária para a empresarial, e mecanismos de apropriação, como a patente, podem vir a criar custos de transação desnecessários, (ETZKOWITZ; LEYDESDORFF, 2000).

\section{A universidade como formadora de recursos humanos e parceira da empresa para inovação - a abordagem de sistemas de inovação}

A abordagem de sistemas de inovação ${ }^{7}$ pressupõe a geração de inovação ${ }^{8}$ como um processo interativo, cujas empresas, locus da inovação, interagem com fornecedores, clientes, concorrentes, universidades, institutos de pesquisa e outros na busca pelo aprendizado de novos conhecimentos. Considera-se ainda o papel do governo e suas instituições no estímulo, na regulação, e na criação do ambiente de inovação, (LUNDVALL et al., 2002; EDQUIST, 2005).

A empresa é o principal ator deste sistema, porém a universidade ganha um papel de relativa importância para a geração de inovações a partir do final do século XX e início do século XXI. Nesse momento, devido ao surgimento de grandes descobertas científicas

\footnotetext{
${ }^{6}$ Vale ressaltar que a importância maior ou menor da patente está diretamente relacionada ao setor de atuação da empresa. Em alguns setores, a patente é o principal instrumento de proteção do conhecimento, como é o caso do setor farmacêutico. A apropriação do conhecimento pela empresa poderá em outros setores ocorrer por outros meios.

${ }^{7}$ Seis características do conceito de sistemas de inovação permitem uma análise do processo de geração de inovação e de seus principais fatores; são elas: (1) foco na inovação e nos processos de aprendizado como fatores endógenos; (2) perspectiva holística e interdisciplinar; (3) abordagem histórica e evolucionária; (4) ênfase na interdependência e na não-linearidade; (5) abrangência de inovações de produto e processo, assim como, subcategorias de tipos de inovação e (6) ênfase no papel das instituições como fatores influentes no processo inovativo, (EDQUIST, 2005).

${ }^{8}$ Entende-se, segundo Edquist (2005), as inovações de produto como novos, ou melhores, bens materiais ou serviços intangíveis e inovações de processo como novas formas de produzir bens e serviços, que podem ser tecnológicas ou organizacionais. De acordo com Lundvall et al. (2002), inovação significa uma nova combinação, que implica em continuidade (elementos existentes) e mudança radical (nova combinação).
}

Econômica-Niterói, v. 20, n. 1, p. 9-29. Junho, 2018 
nas áreas de informação e comunicação e engenharia genética que permitiram aumentar a velocidade de criação de novos conhecimentos, a questão da sua geração entrou na pauta de debates, e com ela é levantada a discussão sobre o papel da universidade para o desenvolvimento econômico.

Entre os autores neo-schumepterianos, duas vertentes - não excludentes e potencialmente complementares - analisam esta atuação da universidade. B-A. Lundvall dá ênfase à questão do aprendizado e às mudanças sentidas na formação dos estudantes da universidade a partir da interação com as empresas, e N. Rosenberg chama atenção para o fim da fronteira nítida, e para a complementaridade entre a pesquisa acadêmica e a industrial.

Lundvall (2007) e Foray e Lundvall (1999) argumentam que o atual desenvolvimento das tecnologias e das inovações é caracterizado por uma economia do aprendizado Lundvall e Johnson (1994), na maioria das vezes interativo, no nível da empresa e dos trabalhadores. Neste novo contexto é posto um novo desafio à universidade: o de fortalecer a capacidade de aprendizado de seus estudantes. Com isso, a maneira como os professores ensinam e como os alunos apreendem torna-se crucial. Este novo desafio estabelece para Lundvall (2007) a principal contribuição da universidade para a geração de inovação, a formação de graduados com boa capacitação em solucionar problemas. No mesmo sentido, Freeman (1994) relembra que Pavitt $(1993)^{9}$ enfatizou que a principal contribuição da pesquisa básica para o setor industrial era indireta, por meio da formação de jovens com novos e valiosos conhecimentos e habilidades.

A contribuição direta através dos artigos publicados, porém, teria uma importância menor. Além deste desafio principal, a universidade teria também que estabelecer apoio ao aprendizado contínuo dos acadêmicos, e forte conexão entre ensino e pesquisa, para que o ensino de sala de aula esteja de acordo com as pesquisas atuais ${ }^{10}$.

Rosenberg (1990), por outro lado, afirma que as empresas realizam pesquisa básica com seus próprios recursos como parte de suas estratégias competitivas e que a principal razão para isto é a busca pelo melhor entendimento de como e para onde conduzir a pesquisa mais aplicada. Neste sentido, não há mais uma fronteira nítida entre a pesquisa realizada na empresa e na universidade. A realização de pesquisa básica ajuda a empresa a tomar decisões estratégicas sobre seu futuro, e funciona também de maneira defensiva, pois permite decisões rápidas e eficientes quando concorrentes introduzem novos produtos no mercado numa direção tecnológica inesperada.

Neste sentido, conforme argumentam Rosenberg e Nelson (1994), Nelson (1990) e Mowery e Sampat (2005), a divisão do trabalho entre pesquisa acadêmica e pesquisa industrial pode não ser tão clara, pois a capacitação gerada a partir da pesquisa básica realizada pelas empresas é essencial para seu relacionamento com o meio acadêmico. Em outras palavras, conforme Cohen e Levinthal (1989), as empresas que desejam ser

\footnotetext{
9 "What do firms learn from basic research?"

${ }^{10}$ Pode-se argumentar aqui que a interação com o setor empresarial também pode ser importante fonte de conhecimento novo e atual para os acadêmicos.
} 
brasileiro

inovadoras e competitivas realizam investimentos internos em P\&D para criação de conhecimento novo, e ampliação da capacidade da empresa de absorção do conhecimento externo. Assim, um melhor aproveitamento pela empresa da pesquisa realizada em universidades, requer capacitação interna da empresa em pesquisa básica, (ROSENBERG, 1990).

Nos setores baseados em ciência, segundo a taxonomia de Pavitt (1984), em especial, a função da universidade vai muito além do fornecimento de recursos humanos qualificados, e a pesquisa acadêmica frequentemente é responsável por iniciar um processo de desenvolvimento e geração de inovações. Freeman (1994) indica que vários estudos já demonstraram a importância para as empresas da interação com atores do sistema de ciência e tecnologia e que a natureza, profundidade, frequência destas interações são altamente específicas à indústria, variando de acordo com a natureza da inovação. Como afirma Lundvall (2002), não é "toda a indústria" que se relacionará com "toda a universidade" de forma contínua para realização de pesquisa. Isto ocorre em setores específicos, tanto da indústria, como da academia.

A aproximação entre empresa-universidade é benéfica para ambas as instituições. A pesquisa acadêmica pode auxiliar a mudança tecnológica por meio da geração da invenção original, ou da versão piloto para o setor industrial desenvolver e comercializar, geralmente em setores ligados à engenharia, ou da promoção de um melhor entendimento e da geração de técnicas para diferentes usos na empresa, o que é mais frequente para a maioria dos setores. Além disso, quanto mais próximo o setor empresarial da academia, mais direcionada às necessidades do primeiro pode ser a qualificação dos estudantes. Por outro lado, ao interagir com empresas, a universidade adquire conhecimento sobre a demanda da sociedade, em geral, e das empresas, em particular, possibilitando a promoção de pesquisas mais próximas destas necessidades, (NELSON, 1990).

Sendo a inovação um processo interativo, o relacionamento com os demais atores do sistema de inovação tornou-se essencial para o sucesso das empresas. Nenhuma empresa hoje, independentemente de seu tamanho, confia somente nas suas competências internas. A formação de redes nacionais e internacionais com as instituições geradoras de conhecimento tornou-se vital para o sucesso das empresas, mas também para a consolidação e desenvolvimento das instituições de conhecimento, como as universidades, (LUNDVALL et al., 2002; EDQUIST, 2005). 


\section{A universidade como ator do desenvolvimento social - uma visão a partir da América Latina}

Alguns autores latino-americanos, que estudam a relação universidade-empresa na América Latina, tais como Arocena e Sutz (2003a, 2005) ${ }^{11}$ e Dagnino (2003), atribuem um papel mais ativo para a universidade no desenvolvimento social ${ }^{12}$. Segundo esses autores, o entendimento do contexto socioeconômico destes países é o primeiro passo para a análise do papel que a universidade pode ter dentro do sistema nacional de inovação. Os altos níveis de desigualdade social e de renda são fortes fatores de influência nesta abordagem, que possui uma grande preocupação com o tema da inclusão social.

Nos tempos atuais, com o conhecimento técnico-científico tendo um papel cada vez mais relevante na economia, a ausência deste conhecimento aparece como um novo agravante das desigualdades pré-existentes. Diversas áreas do planeta sofrem o learning divide Arocena e Sutz (2000, 2003b), ou seja, o atraso de alguns países em suas estruturas de produção e inovação gera uma segregação do conhecimento, fazendo com que estes países se tornem dependentes tecnologicamente dos países mais desenvolvidos. Tais países ficam marginalizados e fora da dinâmica do desenvolvimento econômico, tornando-se cada vez mais dependentes. Um dos fatores que explicam estas deficiências e o consequente fraco desempenho econômico de países em desenvolvimento (PED) é a carência de "espaços de aprendizagem interativa". Estes espaços ${ }^{13}$ são o locus de criação e utilização do conhecimento para solucionar problemas, levando a eventuais inovações, em que diferentes atores são capazes de fortalecer suas capacidades de aprender enquanto interagem na pesquisa para solução de um problema dado.

Segundo Campos (2003), os países menos desenvolvidos não conseguem provocar constantemente spillovers socioeconômicos que sejam capazes de gerar efeitos positivos sobre a economia. Isto ocorre devido ao baixo nível de investimentos e incentivos ao desenvolvimento de processos inovativos, à tradicional importação de tecnologia e ao fato da base de suas vantagens comparativas ser o baixo custo da mão-de-obra e a abundância de recursos naturais. Tais fatores em conjunto dificultam o acompanhamento destes países aos desenvolvimentos de fronteira tecnológica e, consequentemente, da fronteira do conhecimento e do aprendizado.

\footnotetext{
${ }^{11}$ Vale notar que a proposta dos autores uruguaios é também fortemente influenciada pelo papel da Universidad de la República, única universidade pública do Uruguai que possui mais de 70.000 alunos e 7.000 professores num país de 3,3 milhões de habitantes Arocena, Bortagaray e Sutz (2008). A universidade possui um papel diferenciado e altamente importante nas estratégias do país, através da assistência à saúde e a serviços jurídicos para a população mais necessitada, apoio à produção agrária, prestação de serviços sociais, etc.

${ }^{12}$ Apesar de algumas abordagens distintas na América Latina sobre o tema, aqui serão apresentadas somente o enfoque destes três autores.

${ }^{13}$ Tais espaços podem ser altamente institucionalizados formados em organizações ou organizados ad hoc, e dissolvidos após chegar-se ao resultado esperado.
} 

brasileiro

As deficiências nos espaços de aprendizagem e na dinâmica do conhecimento são ao mesmo tempo causas e efeitos da incompletude dos sistemas nacionais de inovação existentes nos PED. De acordo com o Quadro 1, nos PED, em especial na América Latina, a falta de dinâmica inovativa no setor empresarial cria uma demanda de conhecimento escassa, e não estimula a cooperação entre as empresas e com as universidades. As estratégias empresariais, em geral, não demandam conhecimento complexo, o que reduz a necessidade por uma articulação maior com as universidades. Por outro lado, a avaliação acadêmica de modo geral não valoriza a interação com o setor empresarial, concentrandose principalmente no número de horas/aula de cada docente, e a infraestrutura de laboratórios e equipamentos para pesquisa é inadequada Arocena, Bortagaray e Sutz (2008). Sendo assim, a interação entre o meio empresarial e o acadêmico acaba, em sua maioria, por ser realizada apenas por meio de consultorias de forma pontual e com frequência esporádica.

Tabela 1 - Características dos sistemas nacionais de inovação nos países desenvolvidos e em desenvolvimento

\begin{tabular}{|c|c|c|}
\hline & Desenvolvidos & Em desenvolvimento \\
\hline \multicolumn{3}{|l|}{ Comportamento empresarial } \\
\hline $\begin{array}{l}\text { demanda empresarial } \\
\text { de conhecimento }\end{array}$ & Elevada & Escassa \\
\hline cooperação empresarial & Bastante forte & Muita fraca \\
\hline \multicolumn{3}{|l|}{ Demanda de conhecimento } \\
\hline complexidade & $\begin{array}{l}\text { Elevada: universidade } \\
\text { empresarial }\end{array}$ & $\begin{array}{c}\text { Baixa: } \\
\text { universidade } \\
\text { "consultora" }\end{array}$ \\
\hline intensidade & $\begin{array}{l}\text { Elevada: universidade } \\
\text { conectada }\end{array}$ & $\begin{array}{c}\text { Baixa: } \\
\text { Baixa: universidade } \\
\text { isolada }\end{array}$ \\
\hline \multicolumn{3}{|l|}{ Políticas de C\&T } \\
\hline políticas industriais de $\mathrm{C} \& \mathrm{~T}$ & $\begin{array}{c}\text { Reais e implícitas (EUA)/ } \\
\text { Reais e explícitas (Japão e UE) }\end{array}$ & $\begin{array}{l}\text { Aparentes e } \\
\text { variáveis }\end{array}$ \\
\hline $\begin{array}{l}\text { relação dessas políticas } \\
\text { com as políticas econômicas }\end{array}$ & Compatíveis & Contraditórias \\
\hline
\end{tabular}

Fonte: Baseado em Arocena, Bortagaray e Sutz (2008)

A deficiência das políticas explícitas e implícitas de estímulo à inovação piora ainda mais o cenário dos PED. As políticas explícitas ou diretas de CT sofrem escassez de re- 
cursos, o governo não utiliza seu poder de compra de forma a estimular as capacidades nacionais, na maioria das vezes não há qualquer tipo de proteção à indústria nascente e as políticas possuem um curto horizonte de tempo, o que vai contra a natureza das atividades inovativas que necessitam de investimentos de longo prazo para obtenção de resultados. Frequentemente, as políticas de CT seguem uma visão de inovação linear, isto é, além dos problemas apresentados, há a crença de que os avanços em CT se transfiram automaticamente para a inovação. Do lado das políticas implícitas, ou seja, as demais políticas econômicas, principalmente as macroeconômicas, muitas vezes têm um efeito negativo sobre as políticas de CT, pois criam um ambiente hostil para investimentos, principalmente os de longo prazo.

Sendo assim, a distribuição das capacidades científicas e tecnológicas acaba por muito se assemelhar à distribuição da riqueza e da pobreza no mundo, (AROCENA; SUTZ, 2003a). A maior parte dos problemas econômicos e sociais a serem resolvidos se encontra nos PED, sendo que a maior parte do conhecimento é gerado com base nos problemas dos países desenvolvidos (PD).

Arocena, Bortagaray e Sutz (2008) defendem ainda que as questões sociais sejam objeto das políticas científicas e tecnológicas, e que a agenda de pesquisa das universidades seja direcionada mais pelas necessidades gerais da sociedade do que somente pelo interesse de um grupo pequeno da sociedade, as empresas. Adicionalmente, a função extensão (cooperação da universidade com outros atores coletivos para fazer uso social do conhecimento) ganha relevância como terceira missão da universidade, ou seja, a conexão do ensino e da pesquisa com as necessidades sociais localizadas, as quais nem sempre exigem um papel da universidade na fronteira tecnológica.

Dagnino e Thomas (2009), na mesma direção, ressaltam que em termos conceituais, até os anos 1980, entendia-se a atuação da universidade em parceria com a sociedade e o relacionamento universidade-sociedade, mas que depois desta década substitui-se inteiramente o conceito para universidade-empresa. A partir de então, ganham relevância os polos e parques tecnológicos, incubadoras de empresas de base tecnológica, escritórios universitários de transferência de tecnologia e patentes. Estas novas ênfases, segundo os autores, redirecionam o papel das universidades para o desenvolvimento econômico, deixando em segundo plano o desenvolvimento social.

$\mathrm{O}$ foco da agenda de pesquisa no interesse econômico das empresas, segundo estes autores, faria com que a pesquisa e a inovação gerassem mais desigualdade, ao invés de reduzi-la. Dagnino (2003) afirma que a única forma de se ter a comunidade acadêmica e de pesquisa ao lado de um projeto viável economicamente e justo socialmente é chamá-la para a discussão da agenda de pesquisa e, desta forma, tê-la como uma parceira qualificada, engajada e íntegra para apoiar o Estado nas suas políticas científicas e tecnológicas.

Em síntese, a proposta apresentada é que ao invés de as universidades latino-americanas se tornarem uma universidade empresarial periférica, elas deveriam voltar-se para o desenvolvimento social. Ou seja, adquiram uma dimensão maior do que aquela proposta 

brasileiro

pelos neo-schumpeterianos, voltada para a inovação. A agenda de pesquisa e política adotada deveria estar de acordo com as necessidades dos PED para combater o subdesenvolvimento, a pobreza e a miséria, (AROCENA; SUTZ, 2003a).

\section{Discussão}

A partir da combinação das três abordagens apresentadas neste artigo, identificam-se seis formas de atuação da universidade que podem gerar benefícios para a sociedade, seja para a inovação nas empresas, seja para melhorias sociais: (1) formação e treinamento de recursos humanos qualificados, principalmente, mas não exclusivamente, pesquisadores; (2) melhoria da compreensão de tecnologias e avanço do conhecimento; (3) estímulo ao desenvolvimento da PD industrial, complementando-a; (4) desenvolvimento de teorias e métodos para uso posterior pelas empresas, especialmente para setores específicos; (5) criação de empresas; (6) atuação social em áreas básicas como saúde, educação, saneamento, inclusão social, etc.

Vale notar, porém, que estas atuações podem ter maior ou menor viabilidade dependendo do contexto em que as universidades estão inseridas. Os papéis um e dois são resultantes da interpretação da literatura acadêmica sobre as missões da universidade ${ }^{14}$, na consolidação do ensino superior e na formação do sistema científico. Assim, estas atuações estão mais presentes na estrutura da maioria das universidades, ainda que com ênfases diferenciadas dependendo do contexto em que se inserem.

As atuações três, quatro, cinco e seis, por outro lado, foram consideradas como missões das universidades após a segunda revolução acadêmica, em meados do século XX. Estas são mais recentes e são muito dependentes do contexto local, da estrutura da universidade, e dos atores que as cercam. Os PD com base industrial consolidada e direcionada à inovação, marco institucional maduro e universidades de ponta, tendem a criar um melhor ambiente para a atuação da terceira missão das universidades. Na verdade, as abordagens da hélice tríplice e de sistemas de inovação foram elaboradas a partir da identificação do efeito da atuação das universidades no desenvolvimento econômico nos PD.

Os PED que possuem uma base industrial menos estruturada e, em geral, com baixa atividade inovativa, com problemas de desigualdades sociais, e universidades que possuem menos recursos e estrutura para atuar na fronteira do conhecimento, em geral, apresentam mais dificuldades para a atuação das universidades nas atividades três a cinco. Entretanto, o que em geral ocorre ${ }^{15}$ nos PEDs é que as políticas de estímulo à interação empresa-universidade são fortemente influenciadas pela literatura das abordagens que veem a universidade como ator do desenvolvimento econômico, com possibilidade de transferir conhecimentos, licenciar tecnologias, criar empresas e fazer parcerias com em-

\footnotetext{
${ }^{14}$ Introduzida na primeira revolução acadêmica no final do século XIX.

${ }^{15}$ Pelo menos a partir da década de 1980, conforme Dagnino e Thomas (2009).
} 
presas. Todavia, quando os resultados destas ações são analisados, em geral, apresentamse muito incipientes e longe de alcançar os objetivos almejados. Não é por acaso que a abordagem que vê a universidade como ator do desenvolvimento social foi originada na América Latina a partir da identificação da atuação das universidades em PED.

Se analisada a interação empresa-universidade no Brasil, por exemplo, percebe-se que seus os obstáculos são enormes Botelho e Ferreira (2008), Silva e Ruiz (2011), Rocha et al. (2012), Chiarini e Vieira (2012), Schaeffer, Ruffoni e Puffal (2015), Araujo et al. (2015) frente aos poucos resultados de interação encontrados Albuquerque, Silva e Póvoa (2005), Suzigan e Albuquerque (2011), Britto et al. (2012), Rapini, Oliveira e Neto (2013), Gielfi et al. (2017). Eventualmente, um setor ou uma universidade específica pode se destacar frente aos demais, conforme mostra Oliveira (2008), mas o cenário nacional é de interação pontual, esporádica e focada em consultorias e serviços técnicos. É possível perceber claramente as semelhanças com o contexto apresentado pela abordagem da América Latina.

Isto, em grande parte, ocorre pelo perfil pouco inovativo do setor empresarial brasileiro, no qual empresas nacionais realizam baixos esforços inovativos, e empresas multinacionais não veem o Brasil como um centro de desenvolvimento de inovação; como visto, por exemplo, no caso setor farmacêutico brasileiro em Paranhos (2010, 2012).

Os dados da Pesquisa de Inovação (IBGE, 2016) mostram que 38.835 empresas investiram em atividades inovativas no Brasil entre 2012 e 2014, correspondendo a 29,3\% do total de empresas, e que este investimento representou 2,5\% das suas receitas líquidas de vendas. Os fracos esforços levam a resultados também pouco significativos, com $36 \%$ das empresas com introdução de inovações no período. As universidades e institutos de pesquisa têm pouca relevância nos processos inovativos, de acordo com a mesma pesquisa: entre 2012 e 2014, 28,5\% (ou 13.583) das empresas que implementaram inovações utilizaram as universidades como fontes de informação, e apenas 17,4\% (ou 8.305) delas avaliaram a universidade como importante fonte de informação. Entre as empresas com relações de cooperação com outras organizações, 36,7\% (ou 2.677) tiveram a universidade como principal parceira (IBGE, 2016).

Neste cenário empresarial, os resultados da transferência formal de tecnologia das universidades para as empresas são muito pequenos. Os dados do Formulário para Informações sobre a Política de Propriedade Intelectual das instituições científicas e tecnológicas (Formict) mostram que das 264 instituições científicas e tecnológicas (ICTs) com Núcleos de Inovação Tecnológica (NITs) estruturados, 161 tem depósitos de pedidos de patente, mas somente 48 tinham contratos de transferência de tecnologia em 2014. Apesar do crescimento de $60 \%$ e $33 \%$ em relação a 2010 respectivamente, há 216 ICTs $(81 \%$ da amostra) com pedidos de patente, mas sem licenciamento (MCTI 2010 a 2015).

Considerando-se um escopo maior de atividades de parceria, além da transferência formal de tecnologia, os dados do Censo de 2016 do Diretório de Pesquisa do CNPq mostram que, apesar do aumento - 12,7\% em 2010, 26,4\% em 2014 e 33,7\% em 2016 

brasileiro

(CNPQ, 2017) -, somente um terço dos 37.640 grupos de pesquisa do Censo de 2016 declaram interação com empresas ${ }^{16}$.

No tocante à criação de empresas, não há números específicos para incubadoras de empresas de universidades, mas segundo a Associação Nacional de Entidades Promotoras de Empreendimentos Inovadores (Anprotec), há 2.310 empresas incubadas e 2.815 empresas graduadas nas 369 incubadoras em operação no Brasil (ANPROTEC, 2016).

Nas diversas bases de dados para o caso brasileiro, percebe-se que após 13 anos de políticas de estímulo, programas e regulamentação ${ }^{17}$ para ampliar a aproximação entre empresas e universidades não houve muita mudança. Não obstante, os dados apresentados servem apenas para analisar as atuações três, quatro e cinco. Não há dados para analisar o tipo de atuação seis, além de alguns estudos específicos de universidades, como os de Fonseca (2014), que mostra a atuação local dos diversos campi de uma universidade estadual, e Lobo et al. (2013), que discutem a importância da universidade pública na democratização do acesso à educação por meio das plataformas de ensino à distância.

No Brasil, a atuação das universidades com enfoque social foi formalizada no Plano Nacional de Educação (Lei $n^{0} 10.172 / 2001$ ), que estabeleceu a obrigatoriedade de que $10 \%$ do total de créditos exigidos na graduação de nível superior sejam reservados para atuação dos alunos em atividades de extensão. Porém, da mesma forma, a regulação legal não foi suficiente para promover a aproximação das universidades com a sociedade de forma significativa. Há muitas universidades que ainda não conseguiram se adequar a esta realidade, e que não entendem como fazê-lo. Da mesma forma que a parceria universidade-empresa, a relação universidade-sociedade pode não ser adequada para todas as áreas de conhecimento e de atuação da universidade, e será diferente de acordo com o contexto local da universidade.

Desta forma, constata-se que as características específicas dos contextos locais em que as universidades estão inseridas são de extrema relevância para a definição de suas missões. Seja na formação de recursos humanos, na geração de pesquisa ou na atuação para o desenvolvimento social e econômico, é preciso identificar a vocação da universidade e as características do ambiente em que ela está localizada. As ações horizontais que estabelecem missões ou metas uniformes descaracterizam a importância do enraizamento da universidade no contexto em que ela está inserida e seu potencial de atuação.

\footnotetext{
${ }^{16}$ Ressalta-se que a declaração no Diretório é voluntária, atemporal e cumulativa; por exemplo, se o grupo declarou interação em 2010, mas somente este ano ele permanece como grupo com interação em 2016. Este fato leva a uma muito provável superestimação nos dados.

${ }^{17}$ Para maiores detalhes sobre as políticas, programas e o marco legal ver Paranhos, Cataldo e Pinto (2018).
}

Econômica-Niterói, v. 20, n. 1, p. 9-29. Junho, 2018 


\section{Conclusão}

Este artigo fez uma revisão da literatura sobre o relacionamento entre empresas e universidades. Pode-se concluir que as três abordagens sobre os relacionamentos clamam por novas missões para as universidades na questão do desenvolvimento e implicitamente comungam com a visão de que está em curso uma nova forma de produção do conhecimento. Esta nova forma valoriza a sua produção no contexto econômico - hélice tríplice e sistemas de inovação - ou no contexto social - abordagem latino-americana. As diferenças surgem na definição destas novas missões. Em consequência, cada uma das visões sugere formas distintas de intervenções políticas, mas que vão na mesma direção de aumentar a participação entre a missão da universidade e da sociedade, definida de forma ampla ou restrita (mundo econômico).

A partir das abordagens expostas, buscou-se fazer uma análise das estratégias e políticas estabelecidas para aproximação das universidades ao setor empresarial e à sociedade no Brasil. O resultado é que este processo apresenta inadequações, por não ter sido capaz de identificar as características dos atores (empresas e universidades) antes de sua implementação.

Os dados sobre o caso brasileiro mostram que as ações iniciadas em 2004 ainda apresentam em 2018 resultados muito incipientes, em grande parte, pois não se estabeleceu orientações, políticas e marco regulatório baseado no contexto e nas características nacionais. Pode-se dizer que o aparato institucional brasileiro foi construído a partir das abordagens sobre a interação universidade-empresa descolado da realidade nacional. $\mathrm{O}$ estimulo às parcerias entre universidades e empresas não tem como gerar resultados se as empresas não estão investindo ou buscando inovação. Apesar de uma grande e diversa base industrial, as empresas brasileiras desenvolveram-se focadas, em geral, no mercado interno, com foco na redução do custo, e não na criação de novos mercados.

Por outro lado, o sistema científico e tecnológico brasileiro é em grande parte público, e atua sob autogestão, tornando-o moroso, ideológico, amarrado nos limites da administração pública, e com pouca flexibilidade para mudança em sua estrutura. Este perfil dos principais atores é bem diferente dos apresentados nas abordagens teóricas, principalmente, da hélice tripla e de sistemas de inovação, o que não permite que as interações com ICTs sejam pujantes conforme esperado por estas visões.

A implementação deslocada da realidade leva ao estabelecimento dos NITs focados majoritariamente na transferência de tecnologia através de licenciamentos de patentes, sem atentar para o baixo patenteamento brasileiro e à possibilidade de interação através de outras atividades, como a formação de pesquisadores da empresa nos grupos de pesquisa ou de alunos de doutorado com sanduíche nas empresas para complementar sua formação com experiência empresarial.

As políticas de incentivo às parceiras estabeleceram regras e metas fora das características das empresas e das universidades que não foram suficientes para mudá-las e, 

brasileiro

em grande parte, é por este motivo que os resultados continuam pífios. Além disso, estabelecem-se regras seja para a atuação econômica ou social, e espera-se que toda a universidade cumpra as exigências ou expectativas, mas os conhecimentos são diferentes e podem ter efeitos diversos na sociedade, por este motivo, não devem ser tratados igualmente. O que leva à conclusão de que é muito importante considerar o contexto de cada país para se propor políticas baseadas nas abordagens teóricas apresentadas sem a realização de um diagnóstico prévio para verificação de sua viabilidade.

\begin{abstract}
This paper aims to present the characteristics of three approaches on the relationship between companies and universities. In the triple helix model, the university takes the role of commercialization of knowledge; thereby it is an actor of economic development. In the system of innovation approach, the university's role is to train human resources and to be a business partner for innovation. In the Latin-American approach, the university has an important role on the social development, as it would be aligned with peripheral countries necessities. In each one of these approaches universities and companies have different roles and ways of interaction. In Brazil, the incentives to promote university activities for development followed the theoretical frameworks mentioned, but ended up detached from reality. Therefore, the results are incipient and unsatisfactory until now.
\end{abstract}

Keywords: industry-university relationship, triple helix; system of innovation, Latin America, Brazil, (ISI), university-enterprise.

JEL: O30, O32, O38

\title{
Referências bibliográficas
}

ALBUQUERQUE, E. d. M. e.; SILVA, L. A.; PÓVOA, L. Diferenciação intersetorial na interação entre empresas e universidades no Brasil. São Paulo em Perspectiva, v. 19, n. 1, p. 95-104, 3 2005. ISSN 0102-8839. Disponível em: <http://www.scielo.br/scielo.php? script=sci $\_$arttext $\&$ pid $=S 0102-88392005000100008 \& \operatorname{lng}=$ pt\&tlng=pt $>$.

ANPROTEC, A. N. d. E. P. d. E. I. Estudo de impacto econômico: segmento de incubadoras de empresas do Brasil. Brasília: ANPROTEC/SEBRAE, 2016.

ARAUJO, V. d. C. et al. A influência das percepções de benefícios, resultados e dificuldades dos grupos de pesquisa sobre as interações com empresas. Revista Brasileira de Inovação, v. 14, n. 1, p. 77-104, 2015. Disponível em: <https: //scholar.google.com/citations?user=WuEn8PYAAAAJ\&hl=pt-BR\\#d=gs\_md _cita-d\&p=\&u=|\%2Fcitations $\backslash \% 3 F v i e w \backslash$ op $\mid \% 3 D v i e w \backslash$ citation $|\% 26 h||\% 3 D p t-B R|$

Econômica-Niterói, v. 20, n. 1, p. 9-29. Junho, 2018 
\%26user\\%3DWuEn8PYAAAAJ $\% 26$ citation\_for__view\\%3DWuEn8PYAAAAJ \%3AM3ejUd6NZC8Cl\%26tzom\\%3D180>.

AROCENA, R.; BORTAGARAY, I.; SUTZ, J. Reforma universitaria y desarollo. Montevideo: Tradinco, 2008.

AROCENA, R.; SUTZ, J. Interactive Learning Spaces and Development Problems in Latin America. In: DRUID Working Paper 13. [S.1.]: DRUID Working Paper 13, 2000.

AROCENA, R.; SUTZ, J. Subdesarrollo e innovación: Navegando contra el viento. [S.1.]: Cambridge University Press, 2003. Madrid p.

AROCENA, R.; SUTZ, J. Understanding underdevelopment today: new perspectives on NSI. In: The First Globelics Conference. Rio de Janeiro: The First Globelics Conference., 2003.

AROCENA, R.; SUTZ, J. Conhecimento, inovação e aprendizado: sistemas e políticas no Norte e no Sul. In: Conhecimento, sistemas de inovação e desenvolvimento. Rio de Janeiro: Editora UFRJ/Contraponto., 2005.

BOTELHO, A. J.; FERREIRA, F. Financing university-industry relations: university booster or innovation driver. In: SCHWARTZMAN, S. (Ed.). University and Development in Latin America: successful experiences of research centers. Rotterdam: Sense Publishers, 2008. p. 43-72.

BRITTO, J. et al. Competências científico- tecnológicas e cooperação universidadeempresa na saúde. Revista de Saúde Pública, Faculdade de Saúde Pública da Universidade de São Paulo, v. 46, n. suppl 1, p. 41-50, 12 2012. ISSN 00348910. Disponível em: <http://www.scielo.br/scielo.php?script=scil_arttext\&pid= S0034-89102012000700007\&lng=pt\&tlng=pt $>$.

CHIARINI, T.; VIEIRA, K. P. Universidades como produtoras de conhecimento para o desenvolvimento econômico: sistema superior de ensino e as políticas de CT\&amp;I. Revista Brasileira de Economia, v. 66, n. 1, p. 117-132, 3 2012. ISSN 0034-7140. Disponível em: <http://www.scielo.br/scielo.php?script=sci \arttext\&pid= S0034-71402012000100006\&lng=pt\&nrm=iso\&tlng=en $>$.

CNPQ, C. N. d. D. C. e. T. Súmula estatística. 2017. Disponível em: <www.lattes.cnpq. br>.

COHEN, W. M.; LEVINTHAL, D. A. Innovation and Learning: The Two Faces of R \& D. The Economic Journal, WileyRoyal Economic Society, v. 99, n. 397, p. 569, 9 1989. ISSN 00130133. Disponível em: <https://www.jstor.org/stable/2233763?origin=crossref>. 
26 Abordagens teóricas sobre o relacionamento entre empresas e universidades e o cenário brasileiro

DAGNINO, R. A Relação Universidade-Empresa no Brasil e o Argumento da Hélice Tripla. Revista Brasileira de Inovação, v. 2, n. 2, p. 267, 2003. Disponível em: $<$ http://periodicos.sbu.unicamp.br/ojs/index.php/rbi/article/view/8648874>.

DAGNINO, R.; THOMAS, H. Planejamento e Políticas Públicas de Inovação: em direção a um marco de referência latino-americano. Planejamento e Políticas Públicas, v. 0, n. 23, 10 2009. ISSN 01034138. Disponível em: <http://www.en.ipea.gov.br/ppp/ index.php/PPP/article/view/76>.

EDQUIST, C. Systems of Innovation: Perspectives and Challenges. In: FARGERBERG, J.; MOWERY, D.; NELSON, R. (Ed.). The Oxford handbook of innovation. New York: Oxford University Press, 2005. Disponível em: <http://oxfordhandbooks.com/view/10. 1093/oxfordhb/9780199286805.001.0001/oxfordhb-9780199286805-e-7>.

ETZKOWITZ, H. The triple helix of university-industry-government implications for policy and evaluation. [S.1.]: SISTER Working Paper 11, 2002.

ETZKOWITZ, H. Research groups as 'quasi-firms': the invention of the entrepreneurial university. Research Policy, Elsevier Science Publishers B.V. (North-Holland), v. 32, n. 1, p. 109-121, 2003. Disponível em: <https://econpapers.repec.org/article/eeerespol/ v\3a32\_3ay\_3a2003\3ai _3a1\3ap\_3a109-121.htm>.

ETZKOWITZ, H. Hélice tríplice - universidade-indústria-governo: inovação em ação. Porto Alegre: EDIPUCRS, 2009.

ETZKOWITZ, H.; LEYDESDORFF, L. The dynamics of innovation: from National Systems and "Mode 2" to a Triple Helix of university-industry-government relations. Research Policy, North-Holland, v. 29, n. 2, p. 109-123, 2 2000. ISSN 0048-7333. Disponível em: <https://www.sciencedirect.com/science/article/abs/pii/ S0048733399000554>.

FONSECA, Y. Universidade e desenvolvimento local: o caso da Universidade Estadual de Goiás. Tese (Doutorado) — Universidade Federal do Rio de Janeiro, 2014.

FORAY, D.; LUNDVALL, B.-A. The knowledge-based economy: from the economics of knowledge to the learning economy. In: OECD (Ed.). Employment and growth in the knowledge-based economy. [S.1.: s.n.], 1999.

FREEMAN, C. The economics of technical change. Cambridge Journal of Economics, Oxford University Press, v. 18, n. 5, p. 463-514, 10 1994. ISSN 1464-3545. Disponível em: <https://academic.oup.com/cje/article/1671013/The>.

GIBBONS, M. et al. The new production of knowledge: the dynamics of science and research in contemporary societies. London: Sage Publications Inc, 1994.

Econômica-Niterói, v. 20, n. 1, p. 9-29. Junho, 2018 
GIELFI, G. G. et al. University-industry research collaboration in the Brazilian oil industry: the case of Petrobras. Revista Brasileira de Inovação, v. 16, n. 2, p. 325, 8 2017. ISSN 2178-2822. Disponível em: <https://periodicos.sbu.unicamp.br/ojs/index. $\mathrm{php} / \mathrm{rbi} /$ article/view/8650114>.

IBGE, I. B. D. G. E. E. Pesquisa Industrial de Inovação Tecnológica - PINTEC 2014. Rio de Janeiro, 2016.

LEYDESDORFF, L. The knowledge-based economy: modeled, measured, simulated. Florida: Universal Publishers, 2006.

LOBO, M. E. et al. A atuação da universidade no desenvolvimento social: a experiência dos cursos de administração pública no âmbito do PNAP/UAB/UFSC. In: XIII Coloquio de Gestión Universitaria en Américas: Rendimientos académicos y eficacia social de la Universidad. [S.1.]: XIII Coloquio de Gestión Universitaria en Américas: Rendimientos académicos y eficacia social de la Universidad, 2013.

LUNDVALL, B.-A. The university in the learning economy. [S.1.]: DRUID Working Paper 6, 2002.

LUNDVALL, B.-A. Higher education, innovation and economic development. [S.1.], 2007.

LUNDVALL, B.-; JOHNSON, B. The Learning Economy. Journal of Industry Studies, Carfax Publishing Ltd., v. 1, n. 2, p. 23-42, 11 1994. ISSN 1320-6095. Disponível em: $<$ http://www.tandfonline.com/doi/full/10.1080/13662719400000002>.

LUNDVALL, B.- et al. National systems of production, innovation and competence building. Research Policy, North-Holland, v. 31, n. 2, p. 213-231, 2 2002. ISSN 0048-7333. Disponível em: <https://www.sciencedirect.com/science/article/abs/pii/ S0048733301001378>.

MOWERY, D. C.; SAMPAT, B. N. Universities in National Innovation Systems. In: FARGERBERG, J.; MOWERY, D.; NELSON, R. (Ed.). The Oxford handbook of innovation. New York: Oxford University Press, 2005. Disponível em: <http://oxfordhandbooks. com/view/10.1093/oxfordhb/9780199286805.001.0001/oxfordhb-9780199286805-e-8>.

NELSON, R. Capitalism as an engine of progress. In: NELSON, R. (Ed.). The sources of economic growth. Massachusetts: Harvard University Press, 1990.

OLIVEIRA, S. Sobre a interação universidade-empresa no desenvolvimento de software: um estudo de caso no Recife. Tese (Doutorado) - Universidade Federal do Rio de Janeiro, 2008. 
28 Abordagens teóricas sobre o relacionamento entre empresas e universidades e o cenário brasileiro

PARANHOS, J. Interação entre empresas e instituições de ciência e tecnologia no sistema farmacêutico de inovação brasileiro: estrutura, conteúdo e dinâmica. Tese (Doutorado) - Universidade Federal do Rio de Janeiro, 2010.

PARANHOS, J. Interação entre empresas e instituições de ciência e tecnologia: o caso do sistema farmacêutico de inovação brasileiro. Rio de Janeiro: EdUERJ, 2012.

PARANHOS, J.; CATALDO, B.; PINTO, A. C. d. A. Criação, institucionalização e funcionamento dos núcleos de inovação tecnológica no Brasil: características e desafios. Revista Eletrônica de Administração, v. 24, n. 2, p. 253-280, 9 2018. Disponível em: $<$ https://seer.ufrgs.br/read/article/view/84988>.

PAVITT, K. Sectoral patterns of technical change: Towards a taxonomy and a theory. Research Policy, North-Holland, v. 13, n. 6, p. 343-373, 12 1984. Disponível em: $<$ https://www.sciencedirect.com/science/article/abs/pii/0048733384900180>.

RAPINI, M. S.; OLIVEIRA, V. P. d.; NETO, F. C. d. C. e. S. A natureza do financiamento influencia na interação universidade-empresa no Brasil? Revista Brasileira de Inovação, v. 13, n. 1, p. 77, 12 2013. Disponível em: <https: //periodicos.sbu.unicamp.br/ojs/index.php/rbi/article/view/8649072>.

ROCHA, M. de M. et al. Innovation as a Critical Success Factor: an Exploratory Study about the Partnership among University with Pharmaceutical Industry in Brazil. Journal of technology management \& innovation, v. 7, n. 3, p. 148-160, 10 2012. ISSN 0718-2724. Disponível em: <http://www.scielo.cl/scielo.php?script=scil_arttext\&pid= $\mathrm{S} 0718-27242012000300013 \& \operatorname{lng}=\mathrm{en} \& \mathrm{nrm}=\mathrm{iso} \& \operatorname{tlng}=\mathrm{en}>$.

ROSENBERG, N. Why do firms do basic research (with their own money)? Research Policy, North-Holland, v. 19, n. 2, p. 165-174, 4 1990. ISSN 0048-7333. Disponível em: <https://www.sciencedirect.com/science/article/abs/pii/0048733390900469>.

ROSENBERG, N.; NELSON, R. R. American universities and technical advance in industry. Research Policy, North-Holland, v. 23, n. 3, p. 323-348, 5 1994. Disponível em: <https://www.sciencedirect.com/science/article/abs/pii/0048733394900426>.

SCHAEFFER, P. R.; RUFFONI, J.; PUFFAL, D. Razões, benefícios e dificuldades da interação universidade-empresa. Revista Brasileira de Inovação, v. 14, n. 1, p. 105, 2 2015. ISSN 2178-2822. Disponível em: <https://periodicos.sbu.unicamp.br/ojs/index. $\mathrm{php} / \mathrm{rbi} /$ article/view/8649091>.

SILVA, T. C.; RUIZ, R. M. Uma avaliação exploratória do Fundo Setorial da Saúde. Revista Brasileira de Inovação, v. 10, n. 2, p. 343, 11 2011. ISSN 2178-2822. Disponível em: <https://periodicos.sbu.unicamp.br/ojs/index.php/rbi/article/view/8649019>.

Econômica-Niterói, v. 20, n. 1, p. 9-29. Junho, 2018 
SUZIGAN, W.; ALBUQUERQUE, E. d. M. e. The underestimated role of universities for the Brazilian system of innovation. Revista de Economia Política, Centro de Economia Política, v. 31, n. 1, p. 03-30, 3 2011. ISSN 0101-3157. Disponível em: <http: //www.scielo.br/scielo.php?script=scil_arttext\&pid=S0101-31572011000100001\&lng= en\&tlng=en>. 